\title{
Optimization based tuning of fault detection and diagnosis systems for safety critical systems
}

\author{
Daniel Ossmann \\ Institute of System Dynamics and Control, DLR Oberpfaffenhofen, \\ D-82234 Wessling, Germany. (e-mail: daniel.ossmann@dlr.de)
}

\begin{abstract}
The reliable detection of faults located in the control loop of safety critical systems is an important aspect in reducing potential hazards induced by possible faults. Any fault detection and diagnosis system for a safety critical system has to fulfill strong safety specifications which can be expressed in terms of different performance criteria as detection time performance, missed detection rate, or false alarm rate. To satisfy all design requirements in the presence of unknown external signals and parametric uncertainties a tuning of the free parameters in the fault detection and diagnosis system often becomes necessary after the design of residual filters. In this paper an advanced approach to tune these free parameters based on a multi objective parameter optimization setup is presented.
\end{abstract}

Keywords: Fault detection, optimization, safety critical systems.

\section{INTRODUCTION}

Typical challenging problems in today's advanced control systems designs for safety critical systems as cars and aircraft are trying to reduce the human workload, ensuring best handling qualities simultaneously with increased passenger comfort. Despite a high system complexity, the fault tolerant operation of such systems has to be guaranteed over the whole operation range in presence of many possible unexpected events and inherent uncertainties in the operation environment. To increase system autonomy, the industry traditionally uses physical redundancy of e.g. actuators and sensors. However, this hardware-redundancy based fault detection and diagnosis (FDD) approach is becoming increasingly problematic when used in conjunction with the many innovative technical solutions being developed to satisfy the greener imperatives demanded by the society. Hardware redundancy leads to an increased weight and hence, to an increased need of energy driving any movable system. In recent years, to alleviate this fault diagnosis bottleneck, efforts have been invested to develop FDD systems which strongly rely on advanced model based FDD techniques (e.g., Goupil [2010]).

Any FDD system of a safety critical system must fulfill strong performance specifications which can be expressed in terms of several criteria. Typical performance specifications are the detection time performance (DTP), missed detection rate (MDR), and false alarm rate (FAR). Especially for safety critical systems no false alarms and missed detections are required. Commonly in the first phase of the FDD system design a residual filter is designed using wellknown and established design methods. Due to nonlinearities, unknown inputs etc., additional filter functions and thresholds have to be introduced in the FDD system. The available free parameters are then tuned by trial and error and numerous simulations to fulfill these specifications in a second phase of the FDD system design.

Systematic approaches to calculate thresholds for linear models are available in literature (e.g., Gertler [1998]). However, this approach often turns out to be too conservative, leading to a degradation of the FDD system. This paper proposes an alternative approach for the selection of the values of the free parameters, namely based on worst case optimization techniques. Some basic criteria for the FDD system optimization have been presented by the author in previous contributions for different applications (Ossmann and Varga [2011], Varga et al. [2011], Varga and Ossmann [2012]). This paper presents an extended list of criteria together with relevant optimization problems to provide a complete overview of possible optimization setups for FDD systems of safety critical systems.

The main challenge of an optimization based design and analysis of FDD systems is to turn the discussed performance specifications into computable optimization criteria, which is the main contribution of this paper. In section 2 the general structure of a FDD system is given, including the introduction of free parameters for the optimization. In section 3 the performance criteria are presented, while in section 4 the integrated tuning approach using the presented criteria is described. In section 5 the presented methodology is applied to a modern aircraft actuator, which is simulated in a fully nonlinear aircraft model.

\section{FDD SYSTEM STRUCTURE}

In this section the general structure of an FDD system and its subparts is presented. For a reduced complexity a FDD system with a scalar residual signal is discussed. However, the approach presented in the paper can easily be adapted for the MIMO case. A typical FDD system structure for a nonlinear system 


$$
\begin{aligned}
& \dot{\tilde{x}}=F(\tilde{u}, \tilde{x}, \pi, d, f) \\
& \tilde{y}=G(\tilde{u}, \tilde{x}, \pi, d, f),
\end{aligned}
$$

with the control input vector $\tilde{u}$, the state vector $\tilde{x}$, the output vector $\tilde{y}$, the disturbances $d$, the fault vector $f$ and the parameter vector $\pi$ is depicted in Fig. 1 (Gertler [1998]). The underlying system can be a single subsystem as an actuators or sensor, or the whole closed loop system, depending on the location of the faults to be detected and the availability of sensor signals. The FDD system usually includes a residual generator to generate the residual signal $r$, a residual evaluator to compute the evaluation signal $\theta$ and a threshold-based decision making block to generate the binary decision signal $\iota$. The detector uses as inputs the measurement signals $y$ and the control input signals $u$. In what follows a description of the functionality of these blocks is given.

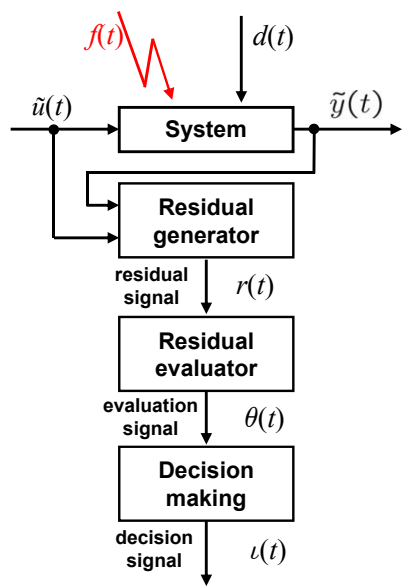

Fig. 1. Typical FDD system structure

\subsection{Residual generator}

A linear residual generator processes the measurable system outputs $\tilde{y}(t)$ and inputs $\tilde{u}(t)$ to generate the residual signals $r(t)$ which serve for decision making on the presence or absence of faults. To design such a filter the fault detection problem has to be solved. Let $\tilde{x}_{0}, \tilde{y}_{0}$ and $\tilde{u}_{0}$ be an equilibrium point corresponding to a fixed $\pi=\pi_{0}$, such that

$$
\begin{aligned}
0 & =F\left(\tilde{u}_{0}, \tilde{x}_{0}, \pi_{0}, 0,0\right) \\
\tilde{y}_{0} & =G\left(\tilde{u}_{0}, \tilde{x}_{0}, \pi_{0}, 0,0\right) .
\end{aligned}
$$

Around this point the linearized additive fault model can be described by the input-output form

$$
y(s)=G_{u}(s) \mathbf{u}(s)+G_{d}(s) \mathbf{d}(s)+G_{f}(s) \mathbf{f}(s),
$$

where $\mathbf{y}(s), \mathbf{u}(s), \mathbf{d}(s)$ and $\mathbf{f}(s)$ are Laplace-transformed vectors of the $p$-dimensional system output vector $y(t)=$ $\tilde{y}-\tilde{y_{0}}, m_{u}$-dimensional input vector $u(t)=\tilde{u}-\tilde{u}_{0}, m_{d}$ dimensional input vector $d(t)$ and $m_{f}$-dimensional input vector $f(t) . G_{u}(s), G_{d}(s)$ and $G_{f}(s)$ are the transferfunction matrices (TFM) from the control inputs to outputs, disturbance inputs to outputs and fault inputs to outputs respectively. The input-output form of a residual generator for this system is given by

$$
\mathbf{r}(s)=Q(s)\left[\begin{array}{l}
\mathbf{y}(s) \\
\mathbf{u}(s)
\end{array}\right],
$$

where $Q(s)$ is in the scalar output case a $1 \times\left(p+m_{u}\right)$ vector. This vector has to be proper and stable to have a physically realizable filter. The residual signal $\mathbf{r}(s)$ generally depends via the system outputs $\mathbf{y}(s)$ of all system inputs $\mathbf{u}(s)$, $\mathbf{d}(s)$ and $\mathbf{f}(s)$. The residual generation system, obtained by replacing $\mathbf{y}(s)$ in (4) by its expression in (3), is given by

$$
\mathbf{r}(s)=R_{u}(s) \mathbf{u}(s)+R_{d}(s) \mathbf{d}(s)+R_{f}(s) \mathbf{f}(s),
$$

where

$$
\left[R_{u}(s)\left|R_{d}(s)\right| R_{f}(s)\right]:=Q(s) G_{e}(s)
$$

with

$$
G_{e}(s):=\left[\begin{array}{c|c|c}
G_{u}(s) & G_{d}(s) & G_{f}(s) \\
1 & 0 & 0
\end{array}\right] .
$$

The choice of $Q(s)$ guarantees an exact decoupling of all control- and disturbance inputs in the equilibrium point for the linear system (3), thus $R_{u}(s)=0$ and $R_{d}(s)=0$, while for $f \neq 0$ the residual signal $r(t)$ is asymptotically bounded, thus $R_{f}(0)=$ const (Varga [2008]). This problem is a widely studied topic in literature. Methods and its applications based on parity spaces (Vanek et al. [2012]), on Kalman filters (Efimov et al. [2012]), on $H_{\infty}$ methods (Marcos [2012]) or nullspace methods (Varga [2008]) amongst others are available. In this paper it is assumed that the fault detection problem is solved for the nominal, linear case and the free parameters are optimized to improve the FDD system performance in case of nonlinearities of the underlying system, unknown disturbances, parameter variations, etc. The exact decoupling of $u$ and $d$ is only achieved for the nominal, linearized system. In all other cases the residual signal $r$ will never be exactly zero. At this point the optimization based approach, described in this paper comes into play.

\subsection{Residual evaluation and decision making}

To decrease the sensitivity of the FDD system in the fault free case, it is often favorable to evaluate the residual signal over a certain period of time. This evaluation of the residual signal requires the computation of a measure of the residual signal energy, for which the 2-norm of the signal is usually an appropriate choice. The so called Narendra signal evaluation scheme as an approximation of the 2 -norm in the form

$$
\theta(t)=\alpha|r(t)|+\beta \int_{0}^{t} e^{-\gamma(t-\tau)}|r(\tau)| d \tau,
$$

can be used. The filter parameters $\alpha \geq 0$ and $\beta \geq 0$ represent weights for instantaneous and long-term values, respectively, and $\gamma>0$ is the forgetting factor. The evaluation signal $\theta(t)$ is compared to a specific threshold $J_{t h}$ in the decision making process to determine the decision signal $\iota$ using the decision logic

$$
\begin{aligned}
& \theta(t)<J_{t h} \Rightarrow \iota(t)=0 \Rightarrow \text { no fault } \\
& \theta(t) \geq J_{t h} \Rightarrow \iota(t)=1 \Rightarrow \text { fault }
\end{aligned}
$$

The appropriate selection of the values of the free parameters $\alpha, \beta$ or $\gamma$, together with an appropriate threshold $J_{t h}$ essentially influences the performance of the FDD system.

\section{FDD PERFORMANCE CRITERIA}

In this section, different relevant performance criteria which can be used for optimal tuning of FDD systems 
but also their a posteriori analysis Ossmann and Varga [2011] are defined. These criteria depend on the values of the free parameters in the architecture of the FDD system. This can be parameters of the residual generator, tolerances and weights of the evaluation filters. We denote these parameters by a vector $\eta$. For the definition of the criteria, we employ the residual evaluation signal $\theta(t)$ which generally depends on the time histories of the signals $u, d, f$, the values of the plant parameters $\pi$ and FDD system parameters $\eta$. Therefore, in what follows, we make this dependence explicit by denoting it as $\theta(u, d, f, \pi, \eta, t)$ and its maximum value over time by $\|\theta(u, d, f, \pi, \eta, t)\|_{\infty}$.

\subsection{Robustness criterion}

For safety critical systems the aim is always to guarantee a minimum or even no false alarms as they may e.g. unnecessarily increase the workload of the human operator and distract him or her from safely operating the system. In the absence of faults we can define the false alarm bound $J_{t h}^{f}$ as

$$
\begin{aligned}
& J_{t h}^{f}(\eta)=\sup _{\substack{\pi \in \Pi, u \in \mathcal{U},}}\|\theta(u, d, 0, \pi, \eta, t)\|_{\infty} . \\
& \begin{array}{l}
u \in \mathcal{U}, \\
d \in \mathcal{D}
\end{array}
\end{aligned}
$$

Instead of arbitrary signals $u$ and $d$, in practical applications it is sufficient to use bounded input signals in a given finite class $\mathcal{U}$ and bounded disturbance signals in a finite class $\mathcal{D}$. The predefined classes $\mathcal{U}$ and $\mathcal{D}$ are typical maneuvers and disturbance signals commonly used to validate the controllers in nonlinear simulations. The vector $\pi$ is assumed to belong to a bounded region $\Pi \subset \mathcal{P}$ of the $n_{p}$-dimensional parameter space $\mathcal{P}$. The value of $J_{t h}^{f}(\eta)$ represents an upper bound for the worst-case magnitude of the residual evaluation signal $\theta$ in the absence of faults. Choosing a threshold $J_{t h}=J_{t h}^{f}$ ensures the absence of false alarms, but also limits the minimum detectable fault amplitude. For a desired finite set $\mathcal{F}$ of bounded fault signals of given magnitudes which are selected to be detected within a required detection time $t_{d}$, the so called detectability bound can be defined as

$$
J_{t h}^{d}(\eta, \Phi)=\inf _{\substack{\pi \in \Pi, f \in \mathcal{F}, t \leq t_{d}}}\|\theta(0,0, f, \pi, \eta, t)\|_{\infty} .
$$

Note that the detection times for different fault signals in $\mathcal{F}$ may even be different. The value of $J_{t h}(\eta, \Phi)$ represents a lower bound for the least magnitude of the residual evaluation signal $\theta$ for fault signals of amplitude $\Phi$. Setting the threshold $J_{t h}=J_{t h}^{d}$ will provide the detection of any fault with an amplitude larger than $\Phi$.

For an ideal design, we have $J_{t h}^{f} \leq J_{t h}^{d}$, thus $J_{t h}^{f} / J_{t h}^{d} \leq$ 1 , enabling a selection of any threshold $J_{t h}^{f} \leq J_{t h} \leq$ $J_{t h}^{d}$, ensuring the detection of the desired minimum fault amplitude and no false alarms. It is reasonable to select an additional gap $\varepsilon \geq 0$ so that $(1+\varepsilon) J_{t h}^{f} \leq J_{t h} \leq(1-$ $\varepsilon) J_{t h}^{d}$ to account for any effects which have not been considered in the design phase, as e.g. modeling errors, unknown disturbances, uncertainties and noise. If for a certain $\eta$ we have that $J_{t h}^{f}>J_{t h}^{d}$, the selection of any $J_{t h}$ leads inevitably to the violation of one of the two defined conditions and either missed detections or false alarms will be the result. In this we suggest to select $J_{t h}=J_{t h}^{f}$ for safety critical systems, ensuring no false alarms, which could unnecessarily distract the operator of the system. In this case missed detections might appear, which is equal to increasing the minimum detectable fault amplitude.

To account for the specifications of missed detections and false alarms we can define the robustness criterion

$$
C_{R B}(\eta):=J_{t h}^{f} / J_{t h}^{d},
$$

directly indicating the robustness of the FDD system. The smaller $C_{R B}$ can be selected, the more robust the decision making process of the FDD system becomes against any affects which have not been considered in the design algorithm.

\subsection{Detection time criterion}

The detection time of a fault signal $f(t)$ is the time moment when the evaluation signal $\theta$ passes for the first time a given threshold $J_{t h}$. The detection time criterion can be defined as the worst-case detection time

$$
C_{D T}\left(\eta, J_{t h}\right)=\sup _{\pi \in \Pi} \min _{t \geq 0}\left\{t \mid \theta(0,0, f, \pi, \eta, t) \geq J_{t h}\right\}
$$

In this definition, $f$ is assumed to be given. When considering a system where the robustness criterion is fulfilled for a given $\eta$, the selection of $J_{t h}=J_{t h}^{f}(1+\varepsilon)$ will lead to the best detection time for a false alarm and missed detection free system.

\subsection{Least detectable fault magnitude criterion}

The fault signals $f$ for different fault scenarios belong to several signal classes $\mathcal{F}_{i}, i=1, \ldots, k$ and their magnitudes within each class are important parameters to define the least magnitudes of detectable faults. For a given detector, we denote by $\Gamma_{f_{\text {min }}}=\left\|f_{\text {min }}\right\|_{\infty}$ the least magnitude of detectable faults, where $f_{\min }(t)$ usually belongs to a given class of faults $\mathcal{F}_{i}$. There are different ways to determine $f_{\text {min }}(t)$ :

If a detection threshold $J_{t h}$ is given, then the corresponding $\Gamma_{f_{\text {min }}}$ results as the least positive value which satisfies the condition

$$
J_{t h}^{d}\left(\eta, \Gamma_{f_{m i n}}\right)=J_{t h},
$$

where the left hand side represents the smallest achievable residual signal corresponding to a fault of amplitude $\Gamma_{f_{m i n}}$. In this case, $\Gamma_{f_{\text {min }}}$ depends on both $\eta$ and $J_{t h}$.

Considering always a robust FDD system $\left(C_{R B} \leq 1\right) \Gamma_{f_{\text {min }}}$ can be determined from the condition that the maximum residual signal which results in the absence of faults is equal to the least achievable residual for the least fault amplitude

$$
J_{t h}^{d}\left(\eta, \Gamma_{f_{\text {min }}}\right)=J_{t h}^{f}(\eta)(1+\varepsilon)=J_{t h},
$$

where $J_{t h}^{f}(\eta)$ is defined in (10). In this case $\Gamma_{f_{m i n}}$ depends only of $\eta$.

In both variants, the determination of $f_{\min }$ involves solving the underlying nonlinear equation, where we can assume that the fault signals are parameterized as $f(t)=$ 
$\Gamma_{f_{\text {min }}}\|\bar{f}(t)\|_{\infty}$, with $\|\bar{f}(t)\|_{\infty}=1$ and $\bar{f}(t) \in \mathcal{F}_{i}$. It follows, that the least detectable fault magnitude criteria

$$
C_{L F}:=\Gamma_{f_{\text {min }}}
$$

is defined only implicitly, via a procedure to compute $\Gamma_{f_{m i n}}$. Explicit approximations of $\Gamma_{f_{m i n}}$ based on appropriate upper bounds evaluation can only be used in a full linear setting.

Minimizing $C_{L F}\left(\eta, J_{t h}\right)$ is equivalent to maximize the sensitivity of the FDD system to faults and thus can be used for optimal tuning of residual generators, but also for tuning the parameters of the FDD system.

\subsection{Optimization based FDD system analysis}

Traditionally, industrial approaches to validate controllers and FDD systems performance rely on statistical analysis of these criteria. In the presence of unknown external signals and parametric uncertainties, finding the worst cases with this approach tends to be difficult and time consuming. Therefore, an optimization approach appears to be better suited if the worst case performance is of interest. An adequate analysis can be achieved using the presented criteria for a fixed set of the parameters $\eta$. This approach offers a fast and efficient method to find deficiencies of the designed FDD system and in this way helps to increase the FDD systems performance. In case of an optimization based design the criteria can be arranged together to determine the best values of the parameters in $\eta$. This will be discussed in the next section.

\section{INTEGRATED TUNING OF FDD SYSTEMS}

With the defined criteria we can formulate several optimization based parameter tuning problems for an integrated design of the FDD system for safety critical systems. In all these problems, we will assume that the input signals $u, d$ and $f$ belong to some finite sets $\mathcal{U}, \mathcal{D}$ and $\mathcal{F}$, respectively, and the parameters $\pi$ have either a continuous variation in a set $\Pi$ or to limit the computational costs in a discrete set $\Pi_{N}$ of parameter values. The sets of inputs can be typical maneuvers or inputs (e.g., step or sine inputs) which have to be considered during the operation of the system. Note, in case of parameterized input signals with rate and amplitude bounds the search domain becomes continuous, while fixed sets lead to a discrete set of input signals, which can be interpreted as a kind of gridding over the possible input signals. From a multitude of optimization problem formulations, we mention only three problems, which are well suited for an integrated parameter tuning in safety critical applications. The primary goal is to optimize the FDD system to ensure that the robustness criterion is fulfilled $C_{R B} \leq 1$ leading no missed detections and false alarms. Hence, the robustness criterion is considered as optimization constraint if other criteria are optimized.

\subsection{Robust synthesis}

An important analysis task which can serve to determine meaningful initial settings of the FDD system parameters in $\eta$ is to assess for the FDD system the lack of false alarms and missed detections in the presence of parametric variations. This analysis can be formulated as an optimization problem to determine

$$
\min _{\eta} C_{R B}(\eta)
$$

so that a selection of $J_{t h}^{f} \leq J_{t h} \leq J_{t h}^{d}$ is possible, if $C_{R B}(\eta) \leq 1$ can be achieved.

\subsection{Minimum detection time optimization}

An optimization-based setting suitable for both detector parameter tuning and for optimal choice of the FDD system parameters is when, besides no false alarms, it is possible to guarantee the required detection time performance (DTP). For this purpose, we can formulate an optimization problem which directly addresses both issues, as follows

$$
\min _{\eta}\left\{C_{D T}\left(\eta, J_{t h}=J_{t h}^{f}\right) \mid C_{R B}(\eta) \leq 1\right\} \text {. }
$$

\subsection{Minimum detectable fault amplitude optimization}

To ensure the fault detectability property of the detector beside the required false alarm free system, it is possible to formulate an optimization problem which directly addresses the issues, as follows

$$
\min _{\eta, J_{t h}}\left\{C_{L F}\left(\eta, J_{t h}\right) \mid C_{R B}(\eta) \leq 1\right\} .
$$

\subsection{Multi-objective optimization based tuning}

Simultaneous requirements on FAR, MDR, DTP, and minimum fault amplitude can be handled by tuning the free parameters of the FDD system using a multi-objective setup, as for example

$$
\min _{\eta}\left\{\nu_{1} C_{D T}\left(\eta, J_{t h}\right) \nu_{2} C_{L F}\left(\eta, J_{t h}\right) \mid C_{R B}(\eta) \leq 1\right\},
$$

where $\nu_{i}, i=1,2$ are suitable weights. Alternatively, strict bounds on detection time can be handled as inequality constraints.

\section{APPLICATION}

In this section an illustrative example with low mathematical complexity is presented to demonstrate the effectiveness of the described optimization based tuning approach. For an elevator control surface and its hydraulic actuator implemented in a modern transport aircraft, a FDD system is set up to be able to detect an actuator jamming during a realistic turn maneuver. To receive realistic actuator inputs as well as a realistic aerodynamic force acting on the actuator, the actuator model and the FDD system is implemented as a subsystem in a nonlinear aircraft model. This enables the possibility, that the free parameters in the FDD systems can be optimized and analyzed in the overall, closed loop aircraft system, using the criteria presented in Section 3 and the integrated tuning approach described in Section 4. A linearized actuator model is considered to illustrate the effectiveness of the presented optimization approach. 


\subsection{Aircraft model and actuator model}

The closed loop aircraft system includes a detailed model of the aerodynamics, a flight mechanical description and a control law. A limited envelope, namely an altitude range of $5500 \mathrm{ft}$, an airspeed range of $30 \mathrm{kts}$, a mass range of $40 \mathrm{t}$ center of gravity range of $10 \%$ around a defined flight condition is considered for the FDD system design. For the estimation of the false alarm bound, a finite set of maneuvers $\mathcal{U}$ is selected. Typical maneuvers used are piloted and auto-piloted flights with various inputs. The actuator jamming is assumed to occur before a coordinated turn maneuver is initiated.

The actuator model represents the dynamics of an hydraulic servo controlled actuator which is widely used in today's aircraft Goupil [2010]. The dynamics are described by a first order model

$$
\dot{\tilde{x}}=k(\pi, \tilde{x}, \dot{\tilde{x}})(u-\tilde{x}),
$$

with the nonlinear gain

$$
k(\pi, \tilde{x}, \dot{\tilde{x}}):=k_{c i} k_{p} \sqrt{\frac{\Delta P(\tilde{x})-\frac{\operatorname{sgn}(\dot{\tilde{x}}) F_{a e r o}(\pi, \tilde{x})+k_{d}(\tilde{x}) \dot{\tilde{x}}^{2}}{S}}{\Delta P_{\text {ref }}}},
$$

where $k_{p}$ is the servo control gain, $k_{c i}$ is a gain to convert an estimated current to a corresponding rod speed, $\Delta P$ is the hydraulic pressure delivered to the actuator, $\Delta P_{r e f}$ is a differential pressure for a fully opened servo-valve (maximum rod speed), $F_{\text {aero }}$ represents the aerodynamic forces at the control surface, $k_{d}(\tilde{x}) \dot{\tilde{x}}^{2}$ represents the estimated servo-control load of the adjacent actuator in damping mode and $S$ is the actuator piston surface area. $\pi$ is a vector of aircraft and flight condition parameters including the angle of attack $\alpha$, the mach number $M a$, the dynamic pressure $p_{d y n}$ and the stabilizer position $i h$.

For a trimmed, level flight condition $\left(\pi=\pi_{0}\right)$ only the stabilizer position $i h$ is used to trim the longitudinal motion of the aircraft with the elevator trim-position $\tilde{x}_{0}=0$ (thus $\dot{\tilde{x}}_{0}=0$ ) leading to

$$
\begin{aligned}
& \Delta P(\tilde{x})=\Delta P(0) \\
& k_{c i}(\tilde{u}, \tilde{x})=k_{c i}(0,0) \\
& k_{d}(\tilde{x}) \dot{\tilde{x}}=0
\end{aligned}
$$

As the influence of the aerodynamic force depends on the moving direction of the actuator, we set $\operatorname{sgn}(\dot{\tilde{x}}) F_{\text {aero }}(\tilde{x}, \pi)$ to a mean value of equal 0 , eliminating the influence of the gain on the $\dot{\tilde{x}}$. With this simplifications a linearized actuator model can be approximated by $\dot{x}=k_{0}(u-x)$ with the linear gain

$$
k_{0}=k_{c i} k_{p} \sqrt{\Delta P(0) / \Delta P_{\text {ref }}},
$$

with the linear gain $k_{0}, x=\tilde{x}$ and $u=\tilde{u}$. With the given hydraulic pressure delivered to the actuator of $\Delta P(0)$, the reference pressure of $\Delta P_{\text {ref }}$, the gains $k_{p}$ and $k_{c i},(22)$ results in a value of $k_{0}=15.7$, which is equivalent to a time constant of $63 \mathrm{~ms}$. This simple model will be used for the design of the residual filter.

Note that the actuator model (21) usually cannot be used directly in an online implementation, as the computational burden is rather high, as the aerodynamic force is often given only as multidimensional table data. An imple- mentable LPV representation of the actuator to cover the whole flight envelope is presented in Varga et al. [2011].

\subsection{Residual generator}

Rewriting the linearized actuator model with the output signal $y=x$ in an input-output form and assuming an input located fault gives

$$
\mathbf{y}(s)=\frac{k_{0}}{s+k_{0}}(\mathbf{u}(s)+\mathbf{f}(s))
$$

For this linear first order model, the residual filter

$$
Q(s)=\left[\frac{a}{k_{0}} \frac{s+k_{0}}{s+a}-\frac{a}{s+a}\right]
$$

can intuitively be designed, where $a$ is an arbitrary positive value specifying the dynamics of the filter. $Q(s)$ guarantees a decoupling of the control inputs in (25) and provides the fault-to-residual transfer function

$$
R_{f}(s)=\frac{a}{s+a} .
$$

Note, that the exact input decoupling is only guaranteed if the real model is equivalent to the linear description (25).

\subsection{Optimization of the free FDD system parameters}

Uncertainties and modeling errors directly influence the residual signal, as the decoupling from the input is not given any more. To minimize these effects, the optimization based approach is used to optimally select the free parameters of the FDD system. The free parameters of the FDD system are the parameters $\alpha, \beta$ and $\gamma$ of the Narendra filter (8). To lower the computational burden the optimization problem is transformed into a multi-case problem Joos [2002] by replacing the continuous search domain $\Pi$ in (10), (11) and (13) by a finite domain $\Pi_{N}=\left\{\pi^{(1)}, \ldots, \pi^{(N)}\right\}$ containing $N=16$ representative grid points. The continuous search domain is considered in an optimization based a posteriori robustness analysis. The optimizations are performed using the optimization environment MOPS (Multi-Objective Parameter Search) of DLR (Joos [2002]). Specifically, the differential evolution global search method is used, which allows to perform many function evaluations in parallel. The results of the optimization are listed in Tab. 1. The nominal case indicates the default situation without optimized parameters, only using the instantaneous weight $\alpha=1$ in (8) to evaluate the residual signal. In this case the robustness criterion is not fulfilled $\left(C_{R B}>1\right)$, inevitably leading to missed detections, when choosing $J_{t h}=J_{t h}^{f}$, required to guarantee no false alarms.

The values for the free parameters of optimization 1 result from an optimization using the robustness criterion $C_{R B}$ solely. In this case the robustness criterion is fulfilled $\left(C_{R B}=0.33\right)$ as its value is below 1 . The evaluation for the detection time criterion $C_{D T}$ in the discrete search domain $\Pi_{N}$ leads to a value of $7.21 \mathrm{~s}$, when setting $J_{t h}=J_{t h}^{f}$. Both criteria values indicate that there may be some room for improvement of the detection time performance at the expense of robustness. In a second run the detection time together with the robustness constraint (18) is optimized. As expected the resulting robustness decreases, while the detection time performance increases. In both optimiza- 
Table 1. Optimization results

\begin{tabular}{ccccc} 
Opt. & criteria & $\alpha ; \beta ; \gamma$ & $C_{R B}\left(\Pi_{N}\right)$ & $C_{D T}\left(\Pi_{N}\right)$ \\
\hline- & none & $1 ; 0 ;-$ & 1.03 & na \\
1 & $C_{R B}$ & $0.01 ; 0.78 ; 0.001$ & 0.33 & $7.21 \mathrm{~s}$ \\
2 & $C_{R B}, C_{D T}$ & $0.01 ; 0.87 ; 0.18$ & 0.44 & $1.59 \mathrm{~s}$
\end{tabular}

tion runs the instantaneous weight $\alpha$ is reduced to its minimum value to reduce the influence of peaks in the residual due to noise. For faster detection time a higher value for the forgetting factor $\gamma$ is required, as low values of the forgetting factor tend to make the FDD system slower.

\subsection{Robustness analysis}

In this section the result obtained by global worst-case search in the continuous search domain $\Pi$ are presented (Tab. 2). As now the continuous search domain is covered by the optimization, the values for the criteria increase but remain in the required bounds. Hence, the selected grid of $N=16$ was chosen adequately to cover the envelope.

Table 2. Worst case search results

\begin{tabular}{ccc} 
Optimization & $C_{R B}(\Pi)$ & $C_{D T}(\Pi)$ \\
\hline- & 1.43 & na \\
1 & 0.34 & $7.8 \mathrm{~s}$ \\
2 & 0.50 & $1.6 \mathrm{~s}$
\end{tabular}

The diagrams in Fig. 2 show the detection times $t_{\text {det }}$ at different points in the envelope as they were calculated in the optimization process to find the worst case of the detection time. The detection times are depicted with different colors, starting from values in cyan $\left(2 \mathrm{~s}<t_{\text {det }} \leq\right.$ $3 \mathrm{~s})$, passing trough yellow $\left(3 \mathrm{~s}<t_{\text {det }} \leq 6 \mathrm{~s}\right)$ to bad values in red $\left(t_{d e t}>6 \mathrm{~s}\right)$. The detection time mainly depends
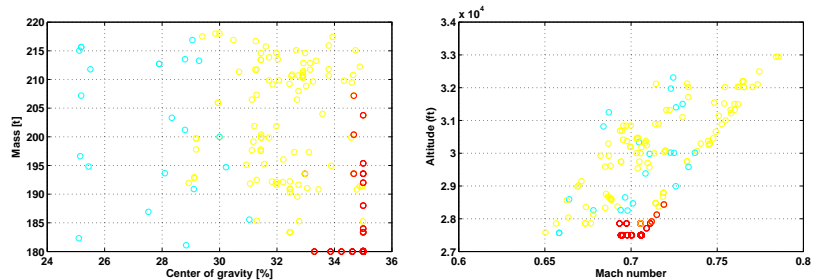

Fig. 2. Detection times in the flight domain using the parameter values from optimization 1
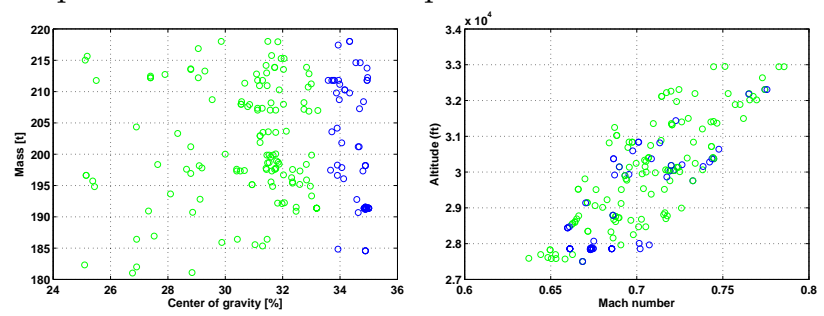

Fig. 3. Detection times in the flight domain using the parameter values from optimization 2

for a fixed set of parameters $\eta$ on the actuator input during the maneuver. The larger the input is, the faster the residual and thus the approximated norm exceed its threshold leading to the detection of the fault. Bad values of the detection time are therefore located at low altitudes in combination with high mach numbers in the flight envelope, since there the increased dynamical pressure and the higher density lead to a decreasing of the required actuator inputs. In the diagrams of Fig. 3 the detection time values for the FDD system with the parameter setting of the second optimization run is depicted, where green points indicate good values $\left(t_{\text {det }} \leq 1 \mathrm{~s}\right)$, and blue still acceptable values $\left(1 \mathrm{~s}<t_{d e t} \leq 2 \mathrm{~s}\right)$ for the detection time. The detection time performance has been improved, so that an actuator jamming is detected in the whole flight envelope within $2 s$.

\section{CONCLUSIONS}

In this paper the optimization based tuning of free parameters in an FDD system has been presented. While the first design of the residual filter can rely on well-known tools linear residual filter design, the followed optimization based tuning process considers any nonlinear elements or inputs of the underlying system. This allows a meaningful selection of the free parameters in the FDD system and avoids the conservativeness of linear approaches or the commonly chosen by trial and error method relying on numerous simulations.

\section{REFERENCES}

D. Efimov, A. Zolghadri, and P. Simon. Fault detection improving by extended Kalman filter adjustment for oscillatory failure case in aircrafts. In Proc. of 4 th European Conference for Aerospace Sciences, St. Pertersburg, Russia, 2011.

J. Gertler. Fault Detection and Diagnosis in Engineering Systems. Marcel Dekker, New York, 1998.

P. Goupil. Oscillatory failure case detection in the A380 electrical flight control system by analytical redundancy. In Control Engineering Practice, 2010, Vol.18, pp. 11101119.

H.-D. Joos. A multi-objective optimization-based software environment for control systems design. In Proc. of CACSD'2002, Glasgow, 2002.

A. Marcos. Application of $H_{\infty}$ fault diagnosis to ADDSAFE benchmark: the control surface jamming case. In Proc. of AIAA Guidance, Navigation and Control Conference, 2011.

D. Ossmann and A. Varga. Optimization based tuning of LPV fault detection filters for civil transport aircraft. In Proc. of 4th European Conference for Aerospace Sciences, St. Pertersburg, Russia, 2011.

B. Vanek, Z. Szabo, A. Edelmayer and J. Bokor. Fault detection of electrical fight control system actuators using parameter dependent estimation. In Proc. of IFAC Symp. SAFEPROCESS'2012, Mexico City, Russia, 2012.

A. Varga. Linear FDI-Techniques and Software Tools. Fault Detection Toolbox V0.8 - Technical Documentation IB 515-08-18, German Aerospace Center (DLR), Institute of Robotics and Mechatronics, 2008.

A. Varga. On parametric solution of fault detection problems. In Proc. IFAC 2011 World Congress, Milano, Italy, 2011.

A. Varga, S. Hecker and D. Ossmann. Diagnosis of actuator faults using LPV-gain scheduling techniques. In Proc. of AIAA Guidance, Navigation, and Control Conference, Portland, Oregon, USA, 2011.

A. Varga and D. Ossmann. LPV-model based identification approach of oscillatory failure cases. In Proc. of IFAC Safeprocess'12, Mexico City, Mexico, 2012. 